\title{
Virtual Teams and Human Work Interaction Design - Learning to Work in and Designing for Virtual Teams
}

\author{
Rikke Orngreen $^{1}$, Torkil Clemmensen ${ }^{2}$, and Annelise Mark Pejtersen ${ }^{3}$ \\ ${ }^{1}$ Assoc. prof., Danish School of Education, Aarhus University \\ ${ }^{2}$ Assoc. prof., Dept. of Informatics, CBS, Denmark \\ ${ }^{3}$ Research Prof., chair of IFIP TC 13
}

\section{Introduction to Theme/Topic}

The boundaries and work processes for how virtual teams interact are undergoing changes, from a tool and stand-alone application orientation, to the use of multiple generic platforms chosen and redesigned to the specific context. These are often at the same time designed both by professional software developers and the individual members of the virtual teams, rather than determined on a single organizational level. There may be no impact of the technology per se on individuals, groups or organizations, as the technology for virtual teams rather enhance situation ambiguity and disrupt existing task-artifact cycles. This ambiguous situation calls for new methods for empirical work analysis and interaction design that can help us understand how organizations, teams and individuals learn to organize, design and work in virtual teams in various networked contexts.

\section{Workshop Objectives and Expected Outcomes}

The objective of this workshop is to raise discussion on both findings from and new methods for studying how people work in and design for virtual teams. The workshop will discuss and present current research on virtual teams as a case of human work interaction design. Topics may include but are not limited to:

- Virtual team cases from public, private and educational institutions

- Formal and informal learning in virtual team design situations

- New methods for empirical analysis and design of collaboration in virtual teams within specific domains and fields of practice

- Studies of virtual team analysis and design activities, e.g. virtual teams performing remote usability testing

- Virtual team design needs with respect to both functionality, usability and pleasurability

- Measures of the time, effort and usefulness spend on 'normal style' work processes as organizations localize and reduce the ambiguity of new virtual team processes.

Through the workshop the participants will help increase awareness of the need for deep understandings of the tasks and processes carried out in virtual team working, as well as the cultural, social and organizational context. Both in practice and in research 
there is an interest in finding, exploring and using new ways of investigating virtual teams learning processes and to investigate how to analyze and transcend findings to design. .

\section{Workshop Organisation and Duration}

This full day workshop begins with presentations (by the workshop organizers and workshop participants) creating a common ground for discussions. Prior to the workshop, participants will be asked to come up with interesting dilemmas, paradoxes or possibilities, based on their work in the position papers. These topics and questions will be the foundation of a more detailed round-table discussion facilitated by the organizers. The workshop will end with a thematic view on the day's discussions.

Participants are required to submit position papers (up to 4 pages), based on the theme and topics listed above. Accepted position papers will be made available to the workshop participants prior to the workshop, at the 13.6 HWID website. The participants will be asked to read the position papers in advance and prepare a couple of questions or remarks to one of the papers (pointed out by the workshop organizers), thus ensuring all papers are not only read thoroughly by the workshop organizers and a least one of the participants, but giving a good basis for dialog.

\section{Target Audience}

The target audience of the workshop is researchers, practitioners and educators, who have experience with virtual teams learning and (re)design processes in human work interaction design. Position papers will be reviewed with respect to their relevance, quality and ability to stimulate discussion. Please send your submission in PDF format, using the standard INTERACT template to: Rikke Orngreen, rior@dpu.dk

\section{Key Organisers of the Workshop}

The organisers of the workshop are members of the 13.6 HWID working group (see http://ilex.cbs.dk/hwid/ and the event-menu on this site for past initiatives). 